\title{
Study the Effect of Seeding Suspension Concentration of DD3R Particles on the Modified Surface of A-Alumina Support for Preparing DD3R Zeolite Membrane with High Quality
}

\author{
Aynaz Meshkat ${ }^{a}$, Mohammad Javad Vaezi ${ }^{b}$, Ali Akbar Babaluo ${ }^{\text {a* }}$ \\ ${ }^{a}$ Nanostructure Materials Research Center, Sahand University of Technology, P.O.Box 51335-1996, Tabriz, Iran \\ ${ }^{b}$ Department of Electrical Engineering, Najafabad Branch, Islamic Azad University, Najafabad, Iran
}

\begin{abstract}
In this study, seeding of DD3R particles as an important step in the synthesis of DD3R membrane with secondary growth method was investigated. Dip-coating method is used for seeding of DD3R particles on the modified surface of $\alpha$-alumina supports. $0.1,0.2$ and $0.3 \mathrm{wt} \%$ aqueous suspensions of the DD3R seeds were prepared and each support was coated three times with dipping time of $240 \mathrm{~s}$. Scanning electron microscopy (SEM) analysis was used for the investigation of the quality of seed layer formed on the support. It is shown that the uniform layer of seeds was formed with $0.2 \mathrm{wt} \%$ seed suspension concentration. DD3R zeolite membrane was synthesized via hydrothermal method on the support seeded with $0.2 \mathrm{wt} \%$ seed suspension concentration. The X-ray diffraction and SEM analysis confirmed the synthesis of uniform DD3R zeolite membrane layer on the support which can be due to the uniform distribution of the DD3R seeds. Single gas permeation tests of N2, CO2 and CH4 were carried out, so that good perm-selectivity for gas mixtures was observed.
\end{abstract}

Keywords:

Seeding;

Seed Suspension Concentration; DD3R;

Zeolite Membrane.

Article History:

Received: 25 July 2017

Accepted: 12 January 2018

\section{1- Introduction}

Natural gas purification (from $\mathrm{CO} 2$ and $\mathrm{N} 2$ ) is so important for using the $\mathrm{CH} 4$ as feed or a fuel. But, conventional approaches for separation of $\mathrm{CO} 2$ and $\mathrm{N} 2$ are expensive units especially at high pressures of natural gas wells [1]. So, Membrane separation by low energy costs and continuous operation can be applied in natural gas processes for separation and purification [2]. Among the membranes, zeolite membranes with well-defined nano-pores have expanded the effort in preparing a membrane with high selectivity and permeance for separation purpose [3, 4]. DD3R zeolite membrane as a new type of zeolite membrane have attractive potential for effective separations of $\mathrm{CO} 2$ and $\mathrm{N} 2$ from CH4 due to its pore channel of $0.36 \mathrm{~nm} \times 0.44 \mathrm{~nm}$ [5]. But in spite of this potential in separation of gases with small molecules, few studies have been done on the synthesis of DD3R zeolite membrane. Because synthesis process of this type of membrane is hurdle and take long time [6]. Therefore, using secondary growth method as a vital technique for accelerating the synthesis of DD3R zeolite membrane with high quality is needed [7, 8]. Effective seeding is one of the essential step to obtain thin and defect free zeolite membranes that prepared with secondary growth method on mesoporous tubular supports. DD3R zeolite membrane was first synthesized by Tomita et al. [9] on macro porous alumina support and reported good separation performance in natural gas purification [9]. Then, some other researchers studied the synthesis of DD3R membrane. But, they reported a poor performance with low quality of membrane layer. According to the literature, the most DD3R membranes with good performance were fabricated by Tomita et al., Himeno et al. and their co-workers at NGK insulator $[5,10]$.

Because of the mentioned importance of seeding, the effective investigation of the different parameter of seeding is necessary [7]. Literature result indicated that the effect of different parameter of seeding was investigated just by Yajima et al. [11]. They studied a seed crystal conditions such as seed crystal average particle diameter and seed crystal weight

\footnotetext{
* CONTACT: A.babaluo@sut.ac.ir

DOI: http://dx.doi.org/10.28991/esj-2018-01127

(C) This is an open access article under the CC-BY license (https://creativecommons.org/licenses/by/4.0/).
} 
per unit area and optimized the values for each parameter. By using these optimized parameters, a uniform membrane layer with low thickness and high permeability was obtained. As the best of our knowledge, the concentration of seeding solution was one of the important parameters in DD3R zeolite membranes synthesis where nobody has yet reviewed.

In the present paper, the $\alpha$-alumina supports modified by TiO2-bohemite were purchased from DPSN Company. The DD3R crystals were coated on these supports via dip-coating method. The quality of the zeolite seed layer formed on the supports was investigated by SEM analysis. Then, the synthesis of DD3R zeolite membrane was carried out on the seeded support with optimized seeding concentration solution via hydrothermal treatment for growing the zeolite layer on the support. The XRD and SEM analyses were used to study the quality of the synthesized membrane layer. To investigate the separation performance of the synthesized DD3R membrane the permeation tests of gases such as N2, $\mathrm{CO} 2$ and $\mathrm{CH} 4$ were carried out.

\section{2- Experimental}

\section{2-1- Materials and Chemicals}

The chemicals and materials used in this work were 1-adamantylamine (ADA, 97\% Sigma-Aldrich), ethylene diamine (EDA, 99.5\%, Merck), tetramethyl orthosilicate (TMOS, 98\%, Merck) and deionized water. $\mathrm{N}_{2}, \mathrm{CO}_{2}$ and $\mathrm{CH}_{4}$ with high purity were used for membrane permeation tests.

\section{2-2- Morphology and Crystallinity}

Scanning electron microscopy (SEM) was used for examine the microstructure and morphology of the seeded support and DD3R membrane and also to investigate the effective thickness. Surface roughness of the modified support was determined by Atomic force microscopy (AFM) analysis. XRD pattern of the synthesized DD3R zeolite membrane was collected by X-Ray diffract meter with $\mathrm{Cu}$ K-X-radiation to confirm the crystal phase.

\section{2-3- DD3R Powder Synthesis}

For preparing the reactant mixture, the amount of 1-adamantane amine was dissolved in ethylene diamine. Then, the calculated amount of deionized water was added to the solution under vigorous mixing for $1 \mathrm{~h}$. After that, the solution was heated at $95^{\circ} \mathrm{C}$ under mixing for $1 \mathrm{~h}$. The obtained solution was cooled and tetramethyl orthosilicate as silica source was slowly added. The resulting solution was heated again for $3 \mathrm{~h}$ at $95^{\circ} \mathrm{C}$. The final solution with molar ratio of 100 $\mathrm{SiO}_{2}: 11240 \mathrm{H}_{2} \mathrm{O}: 47$ 1-ADA :404 EDA was poured into Teflon autoclave and followed by hydrothermal conditions $\left(160^{\circ} \mathrm{C}\right.$ with $\left.60 \mathrm{rpm}\right)$. The synthesized powder was centrifuged with deionized water to decrease the $\mathrm{pH}$ of the powder to neutral.

\section{2-4- Seeding the Support}

The supports were washed to remove dusts and impurities. Thereafter, they were dried at $90{ }^{\circ} \mathrm{C}$ for $3 \mathrm{~h}$. After preparing the DD3R seeds and the supports, the seeds were coated on the surface of supports via dip-coating method by the following procedure. Three different concentration of DD3R seed solutions $(0.1,0.2$ and 0.3 wt $\%$ aqueous suspensions of the seeds) were prepared. Suspensions were stirred for $24 \mathrm{~h}$ at room temperature and followed by sonication to better dispersion and prevent the formation of aggregates. The prepared supports were immersed vertically into seeds suspensions for $240 \mathrm{~s}$ as dipping time and repeated for three times to obtain the high coverage of the support with DD3R crystals. After each dipping, the seeded supports were dried at $90{ }^{\circ} \mathrm{C}$ for $2 \mathrm{~h}$.

\section{2-5- DD3R Membrane Synthesis}

The DD3R tubular membrane was synthesized by secondary growth method with hydrothermal treatment. The synthesis solution for membrane layer was prepared according to the section 1.3. Then, the resulting solution was poured into the Teflon autoclaves where the seeded support was placed in it and heated in an oven at $160{ }^{\circ} \mathrm{C}$. The synthesized membrane was washed with deionized water until the $\mathrm{pH}$ of the washing water decreased to neutral. Before calcination in a furnace at $500{ }^{\circ} \mathrm{C}$ for $50 \mathrm{~h}$, the membrane was dried at $45^{\circ} \mathrm{C}$ for $48 \mathrm{~h}$.

\section{2-6- Single Gas Permeation Test}

$\mathrm{N}_{2}$ Gas permeation test was carried out as the primary test of the membrane layer before removal of structure directing agent (SDA). Because the SDA molecules were filled the zeolitic pores of DD3R. Therefore, a completely defect-free membrane has no permeance to any gases. The gas permeation test contains two sections including; feeding of the gases and separation of the gases (permeation cell). The membrane was sealed with O-ring in a stainless steel module. A schematic of the membrane module has shown in Figure 1. After the calcination process, the permeation test of the calcined membrane was carried out by using constant pressure method. A single gas of $\mathrm{N}_{2}, \mathrm{CO}_{2}$ and $\mathrm{CH}_{4}$ was used as feed gases and fed outside of the zeolite membrane and the permeated flux of gas through the membrane was measured with a soap film flow meter. 


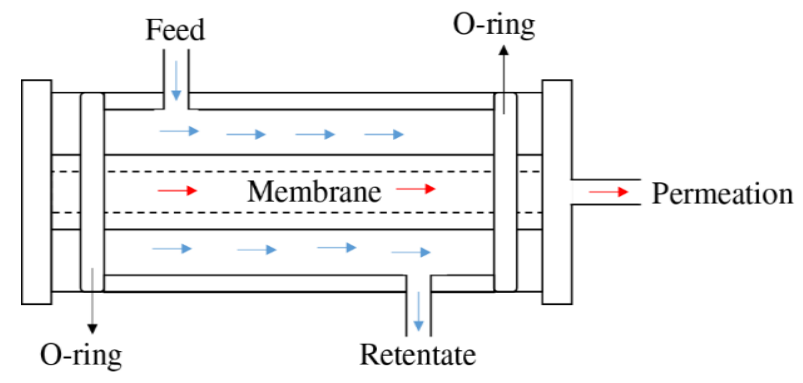

\section{3- Results and Discussion}

Figure 1. Schematic of membrane module.

Figure 2. shows the SEM analysis of $\alpha$-alumina supports before and after modification. As smooth and uniform surface of supports is better for seeding, surface roughness of modified supports was measured by AFM analysis. Figure 3. illustrated the AFM analysis of the modified supports. By using Nano-surf software the average surface roughness for $\mathrm{TiO}_{2}$-bohemite as top layer was calculated $\approx 60 \mathrm{~nm}$.
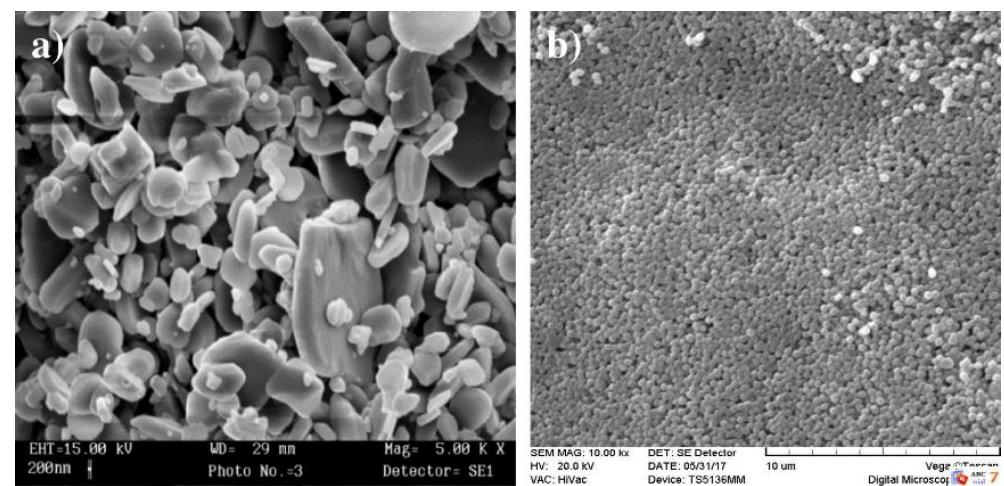

Figure 2. SEM images of $\alpha$-alumina supports (a) before and (b) after modification by $\mathrm{TiO}_{2}$-bohemit.
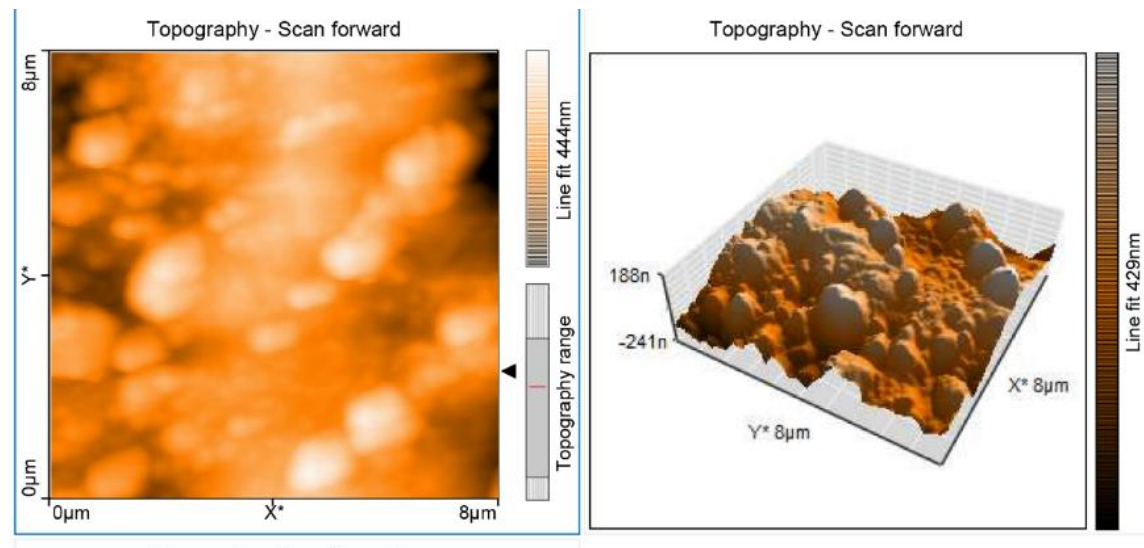

Topography - Scan forward

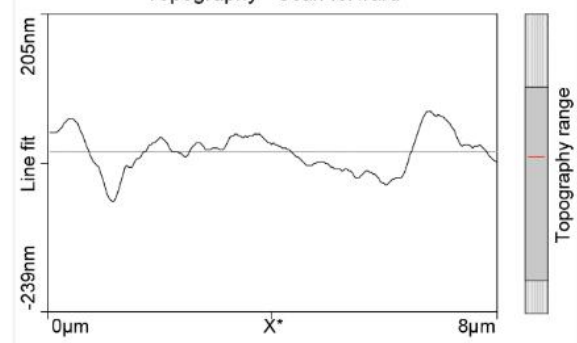

Figure 3. AFM analysis of the modified support.

Figure 4. shows the SEM images of the seeded support with $0.1,0.2$ and 0.3 wt $\%$ concentration of DD3R zeolite seeds solution. As shown in Figure 4 (a) and (c), there is a poor coverage of the seeds on the surface and some areas are without seed coverage. Thus, the formed layer is not uniform. According to Figure 4 (b), it is clear that an appropriate coverage for support has been obtained. These results indicate that seeding the support with $0.2 \mathrm{wt} \%$ concentration of seeds solution leads to the best and uniform coverage on the support. 


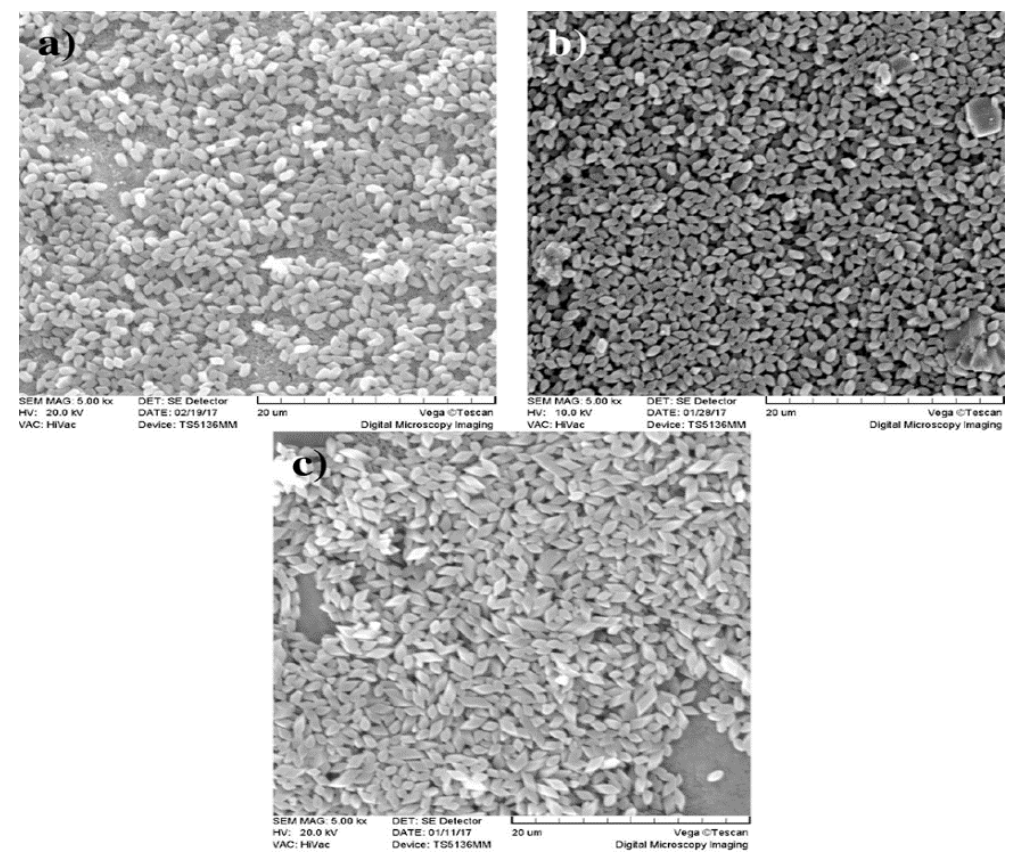

Figure 4. SEM images of the surface of seeded support with a) $0.1 \mathrm{wt} \%$ b) $0.2 \mathrm{wt} \%$ c) $0.3 \mathrm{wt} \%$ concentration of seeds solution.

Figure 5. depicts the XRD pattern of DD3R zeolite membrane obtained by hydrothermal treatment. As can be seen, all the major peaks of DD3R zeolite membrane has completely been formed. This pattern confirmed that the DD3R zeolite membrane prepared from DD3R seeds with $0.2 \mathrm{wt} \%$ concentration of zeolite seeds solution was formed on the modified support.

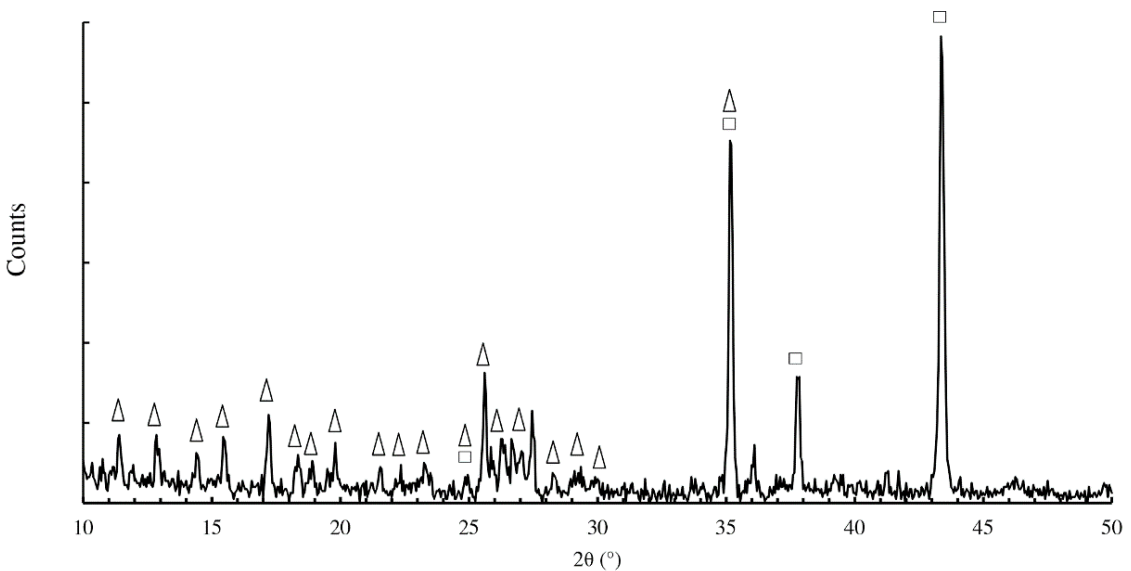

Figure 5. XRD pattern of the synthesized DD3R zeolite membrane.

Figure 6. shows the cross section and top view of the surface of the synthesized DD3R membrane. For the synthesis of this membrane, $0.2 \mathrm{wt} \%$ concentration of the seeds solution has been used. As can be seen, the uniform zeolite layer was formed on the surface support and this is due to the uniform distribution of the DD3R seeds on the modified support surface during seeding step. According to cross sectional SEM image, the thickness of DD3R layer is around $20 \mu \mathrm{m}$.
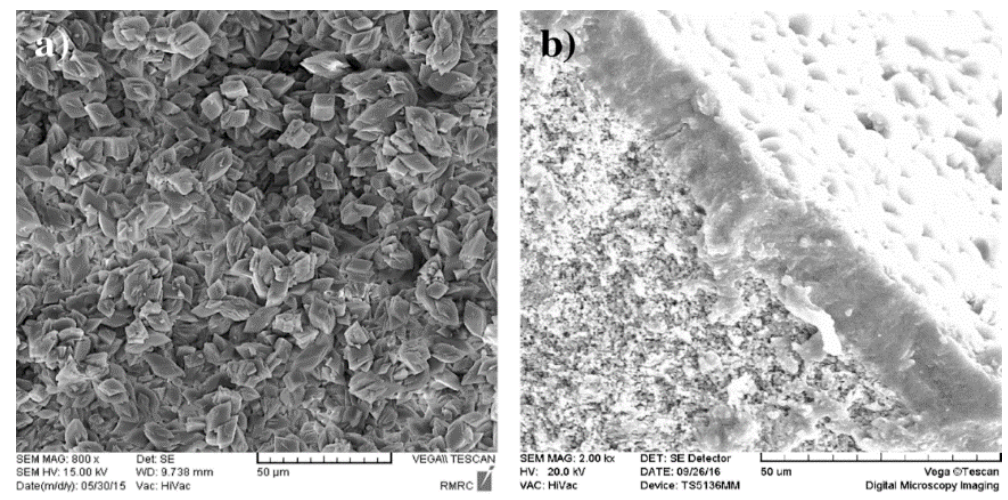

Figure 6. SEM images of the synthesized DD3R membrane (a) surface and (b) cross section. 
Figure 7. showed the perm-selectivity of the synthesized DD3R membrane as a function of the feed pressure. As can be seen, the perm-selectivity of $\mathrm{N}_{2}$ from $\mathrm{CH}_{4}$ decreases at lower pressure range of 1 to 2 bar and remains approximately constant from 2 to 5 bar. Decreasing in perm-selectivity related to existence of some non zeolitic pores while these pores roles efficiently increase by increasing the pressure. This trend is also the same for the perm-selectivity of $\mathrm{CO}_{2}$ from $\mathrm{CH}_{4}$. Also, the high perm-selectivity of $\mathrm{CO}_{2}$ in comparison with $\mathrm{N}_{2}$ is due to strongly adsorption of $\mathrm{CO}_{2}$ on the DD3R zeolite over $\mathrm{N}_{2}$ in the separation of natural gas impurities.

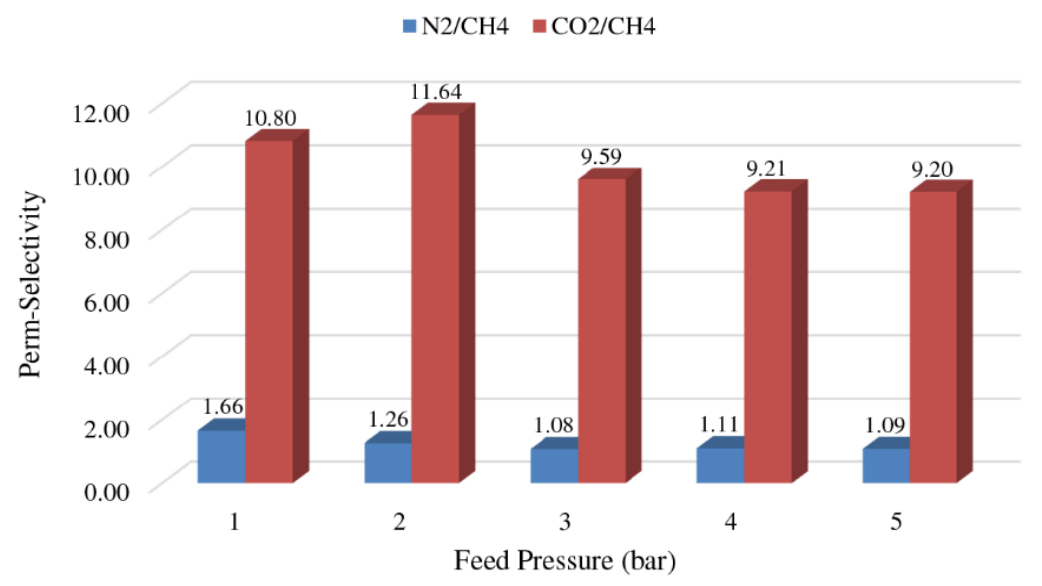

Figure 7. Comparison of the perm-selectivity of the $\mathrm{CO}_{2} / \mathrm{CH}_{4}$ and $\mathrm{N}_{2} / \mathrm{CH}_{4}$ through the DD3R membrane as a function of feed pressure at room temperature.

\section{4- Conclusion}

In this work, it is concluded that uniform DD3R zeolite seed layer can be formed on the modified surface of $\alpha$ alumina support using dip coating method with $0.2 \mathrm{wt} \%$ seed suspension concentration. Synthesis of DD3R membrane layer on the seeded support with uniform seed layer induced formation of uniform zeolite layer. The single gas permeation results showed a good performance in separation of $\mathrm{CO}_{2}$ from $\mathrm{CH}_{4}$. This synthesis procedure with optimized seed suspension concentration could have very promising results to achieve the selective DD3R zeolite membrane for natural gas purification.

\section{5- References}

[1] Wu, Ting, Merritt C. Diaz, Yihong Zheng, Rongfei Zhou, Hans H. Funke, John L. Falconer, and Richard D. Noble. "Influence of Propane on CO2/CH4 and N2/CH4 Separations in CHA Zeolite Membranes." Journal of Membrane Science 473 (January 2015): 201-209. doi:10.1016/j.memsci.2014.09.021.

[2] Den Exter, M.J., J.C. Jansen, and H. van Bekkum. "Separation of Permanent Gases on the All-Silica 8-Ring Clathrasil DD3R." Zeolites and Related Microporous Materials: State of the Art 1994 - Proceedings of the 10th International Zeolite Conference, Garmisch-Partenkirchen, Germany, 17-22 July 1994 (1994): 1159-1166. doi:10.1016/s0167-2991(08)63653-8.

[3] Van den Bergh, J., W. Zhu, J. Gascon, J.A. Moulijn, and F. Kapteijn. "Separation and Permeation Characteristics of a DD3R Zeolite Membrane.” Journal of Membrane Science 316, no. 1-2 (May 2008): 35-45. doi:10.1016/j.memsci.2007.12.051.

[4] Den Exter, M.J., J.C. Jansen, H. van Bekkum, and A. Zikánova. "Synthesis and Characterization of the All-Silica 8-Ring Clathrasil DD3R Comparison of Adsorption Properties with the Hydrophilic Zeolite A.” Zeolites 19, no. 5-6 (November 1997): 353-358. doi:10.1016/s0144-2449(97)00115-2.

[5] Yang, S., Cao, Z., Arvanitis, A., Sun, X., Xu, Z., Dong, J. (2016). DDR-type zeolite membrane synthesis, modification and gas permeation studies. Journal of Membrane Science, vol. 505, p. 194-204.

[6] Mubashir, Muhammad, Yin Fong Yeong, and Kok Keong Lau. "Ultrasonic-Assisted Secondary Growth of Deca-Dodecasil 3 Rhombohedral (DD3R) Membrane and Its Process Optimization Studies in CO2/CH4 Separation Using Response Surface Methodology.” Journal of Natural Gas Science and Engineering 30 (March 2016): 50-63. doi:10.1016/j.jngse.2016.01.015.

[7] Xiao, Wei, Zhan Chen, Liang Zhou, Jianhua Yang, Jinming Lu, and Jinqu Wang. "A Simple Seeding Method for MFI Zeolite Membrane Synthesis on Macroporous Support by Microwave Heating." Microporous and Mesoporous Materials 142, no. 1 (June 2011): 154-160. doi:10.1016/j.micromeso.2010.11.029.

[8] Liu, Yanmei, Zhanzhao Yang, Congli Yu, Xuehong Gu, and Nanping Xu. "Effect of Seeding Methods on Growth of NaA Zeolite Membranes." Microporous and Mesoporous Materials 143, no. 2-3 (September 2011): 348-356. doi:10.1016/j.micromeso.2011.03.016.

[9] Tomita, Toshihiro, Kunio Nakayama, and Hitoshi Sakai. "Gas Separation Characteristics of DDR Type Zeolite Membrane." Microporous and Mesoporous Materials 68, no. 1-3 (March 2004): 71-75. doi:10.1016/j.micromeso.2003.11.016. 
[10] Himeno, Shuji, Toshihiro Tomita, Kenji Suzuki, Kunio Nakayama, Kenji Yajima, and Shuichi Yoshida. "Synthesis and Permeation Properties of a DDR-Type Zeolite Membrane for Separation of CO2/CH4Gaseous Mixtures." Industrial \& Engineering Chemistry Research 46, no. 21 (October 2007): 6989-6997. doi:10.1021/ie061682n.

[11] Yajima, K., Nakayama, K. (2009). Process for producing DDR type zeolite membrane. Patent No. : US 2009/0011926 A1. 